\title{
El impacto de los programas y la condicionalidad del FMI en los derechos laborales
}

\author{
The Impact of IMF Programs and \\ Conditionality on Labor Rights
}

\author{
Su-Hyun Lee ${ }^{1}$ \\ Universidad Tecnológica de Nanyang, Singapur \\ Byungwon $\mathrm{Woo}^{2} \uparrow$ \\ Universidad Yonsei, Corea del Sur
}

Revista Derechos en Acción ISSN 2525-1678/ e-ISSN 2525-1686

Año 6/NNo 18 Verano 2020-2021 (21 diciembre a 20 marzo), 435-478

DOl: https://doi.org/10.24215/25251678e488

Resumen: ¿Los programas y las condiciones del Fondo Monetario Internacional (FMI) perjudican los derechos laborales de los países que participan de los programas? Los críticos del FMI sostienen que participar de los programas de dicha institución es perjudicial a los derechos laborales, ya que el FMI intenta imponer políticas laborales proempresa a través de condicionalidades en ocasiones en las que un país no tiene otra opción que participar de un programa. En contraposición, la gestión del FMI ha reforzado su discurso en cuanto a la manera en que los programas del FMI intentan impulsar el crecimiento inclusivo, a través del

\footnotetext{
1 Profesora asistente. Facultad de Estudios Internacionales S. Rajaratnam. Universidad Tecnológica de Nanyang, Singapur (RSIS/NTU). Contacto: isshlee@ntu.edu.sg

Su Hyun Lee agradece a la Facultad de Estudios Internacionales S. Rajaratnam de la Universidad Tecnológica de Nanyang (RSIS/NTU, por sus siglas en inglés) por su generosa ayuda económica.

2 Profesor asociado. Departamento de Ciencias Políticas y Estudios Internacionales, Universidad Yonsei, Corea del Sur. Contacto: bwwoo@yonsei.ac.kr

El autor de contacto Byungwon Woo agradece la generosa ayuda económica recibida de parte de la Universidad Yonsei a través del Fondo de Investigación de 2019-22-0017.
} 
empleo productivo y la protección de la población más vulnerable dentro de cada país. En este artículo, sostenemos que, si bien es posible que el FMI pueda haber intentado que sus programas resulten beneficiosos para los/as trabajadores/as, sus esfuerzos no son suficientes para revertir los efectos negativos que tienen sobre los derechos laborales, tanto a corto como a largo plazo. Esto no solo se debe a los cambios directos en las políticas que se exigen en los programas del FMI, sino también a las consecuencias indirectas de la liberalización económica. Demostramos que los programas del FMl que imponen condiciones laborales y económicas más estrictas causan mayor cantidad de efectos perjudiciales, tanto en derechos laborales de iure como en prácticas laborales de facto. Asimismo, nuestro análisis destaca la importancia de la política local y expone que los efectos negativos generales de los programas del FMl pueden ser compensados cuando el gobierno participante es de izquierda o cuando un país adopta un sistema de representación proporcional.

Abstract: Do International Monetary Fund (IMF) programs and conditions make harm to labor rights in program-participating countries? Critics of the IMF contend that participation in an IMF program is detrimental to workers' rights as the IMF tries to impose pro-business labor policies via conditionality when a country has no choice but to participate in a program. Countering the criticisms, the IMF management has stepped up its rhetoric on how IMF programs try to promote inclusive growth, providing productive employment, and protecting the most vulnerable population within a country. In this paper, we argue that while the IMF might have tried to make IMF programs more beneficial to workers, those efforts are too little to reverse the overall negative effects on labor rights in both short term and long term. This is not only because of direct policy changes mandated in IMF programs but also because of indirect consequences of liberalization of the economy. We demonstrate that IMF programs with stricter labor-market conditions have more detrimental effects on both de jure labor rights and de facto labor practices. Our analysis also highlights the importance of domestic politics and shows that negative overall effects of IMF programs can be offset when a participating government is leftist or when a country adopts a proportional representation system. 


\section{Introducción}

¿Los programas y las condiciones del Fondo Monetario Internacional (FMI) perjudican los derechos laborales de los países que participan de los programas? Los críticos del FMI asienten enfáticamente y sostienen que el FMI intenta imponer políticas laborales proempresa a través de condicionalidades en ocasiones en las que un país no tiene más opción que participar de un programa. En contraposición, la gestión del FMI ha reforzado su discurso en cuanto a la manera en la que los programas del FMI intentan impulsar el crecimiento inclusivo, a través del empleo productivo y la protección de la población más vulnerable dentro de cada país. ${ }^{3}$

En este artículo sostenemos que, si bien es posible que el FMI puede haber intentado que sus programas resulten beneficiosos para los/as trabajadores/as, sus esfuerzos no son suficientes para revertir los efectos negativos generales que los programas del Fondo y sus condicionalidades causan sobre los derechos laborales, tanto a corto como a largo plazo. Esto no solo se debe a los cambios directos en las políticas que se exigen en los programas del FMI, sino también a las consecuencias indirectas de la liberalización económica. Por medio del uso de una base de datos sobre derechos laborales y otra sobre las condicionalidades del FMI en el mercado laboral, demostramos que los programas del Fondo que imponen condiciones laborales y económicas más estrictas causan mayor cantidad de efectos perjudiciales, tanto en derechos laborales de iure como en prácticas laborales de facto.

Sin embargo, los efectos negativos generales de los programas del FMI no son uniformes en todos los países. A raíz de esto, demostramos teórica y empíricamente que los efectos negativos pueden ser compensados ante la presencia de ciertos

3 Christine Lagarde. directora gerente del Fondo Monetario Internacional, Challenges of Job-Rich and Inclusive Growth (8 de octubre de 2014) https://www.imf.org/en/News/ Articles/2015/09/28/04/53/sp100814. 
factores políticos locales. Uno de esos factores es la inclinación política del gobierno participante: los gobiernos de izquierda, cuya subsistencia política gira en torno al respaldo a los/as trabajadores/as, son propensos a intervenir en la implementación de la condicionalidad con el fin de mitigar sus efectos negativos sobre los/as trabajadores/as. Además, cuando el sistema electoral favorece la representación política de los/as trabajadores/as, en especial cuando hay una representación proporcional, aporta protección contra el daño que la condicionalidad del FMI ocasiona sobre los/as trabajadores/as.

Este artículo se encuentra organizado de la siguiente manera: en la próxima sección repasamos brevemente los estudios existentes sobre la manera en que los programas del FMI afectan a los/as trabajadores/as y los derechos laborales. A continuación, presentamos nuestro argumento teórico y creamos un conjunto de hipótesis comprobables. En las siguientes dos secciones debatimos nuestra estrategia empírica y presentamos y analizamos los principales resultados. A modo de conclusión, debatimos las implicancias sobre las políticas y los planes a futuro.

\section{Análisis bibliográfico}

A pesar de la relevancia política del conflicto entre el FMI y los/as trabajadores/as en los países que participan de los programas, solo se han llevado a cabo unos pocos estudios académicos sobre el impacto que dicha participación tiene en los derechos de los/as trabajadores/as.

Pastor Jr. aporta una de las primeras evaluaciones empíricas sobre el efecto del FMI en el trabajo. ${ }^{4}$ Teoriza que "el deseo del FMI por asegurar la cooperación de las élites locales puede conducirlo a diseñar programas que ubican la carga del ajuste en los

\footnotetext{
4 Manuel Pastor Jr., "The Effects of IMF Programs in the Third World: Debates and Evidence from Latin America", 2 World Development, 1987, Vol. 15, 249-262.
} 
trabajadores y otras clases populares". ${ }^{5}$ Por lo tanto, prevé que los programas del FMI afecten negativamente este sector de la sociedad. Su análisis empírico revela que el curso de los programas del FMI redujo de manera significativa la participación del trabajo en el ingreso de 18 países latinoamericanos entre 1965 y 1981. De manera similar, Weeks atribuye el importante deterioro de los derechos de los/as trabajadores/as y el aumento del desempleo a la liberalización y desregulación de las políticas exigidas por el FMI. Para hacer esta afirmación, Weeks se basa en distintos análisis de las condiciones del mercado laboral en países latinoamericanos entre 1970 y $1998 .{ }^{6}$ Vreeland también considera que la participación en programas del FMI tiene un efecto negativo en la distribución del ingreso y disminuye la participación del trabajo en el ingreso que se genera a partir de la industria, incluso cuando se controlan factores observables y no observables que influyen en la selección para participar de los programas del Fondo. ${ }^{7}$

El estudio de Oberdabernig sobre el impacto de los programas del FMI en la pobreza y la desigualdad presenta resultados más sutiles. Concluye que los programas del FMI afectaron de manera negativa la pobreza y la desigualdad a corto plazo entre 1982-2009. Sin embargo, tales efectos se ven revertidos cuando el cálculo se restringe al subperíodo 2000-2009, durante el cual el Fondo incluyó la reducción de la pobreza en su agenda. En ese caso, los efectos contemporáneos de la participación en los programas del FMI son, en términos estadísticos, positivos y significativos, al reducirse las brechas de pobreza y los índices de Gini. ${ }^{8}$ Blanton et al. utilizan un conjunto de datos

\footnotetext{
5 Ibíd., p. 258.

6 Weeks, John. "Wages, Employment and Workers' Rights in Latin America, 1970-98", 2 International Labour Review, 1999, Vol. 138, 151-169.

7 James Raymond Vreeland, "The Effect of IMF Programs on Labor", 1 World Development, 2002, Vol. 30, 121-139.

8 Doris A. Oberdabernig, "Revisiting the Effects of IMF Programs on Poverty and Inequality", World Development, 2013, Vol. 46, 113-142.
} 
sobre derechos de los/as trabajadores/as para examinar de qué manera los programas de las Instituciones Financieras Internacionales (IFI) afectan los derechos laborales y demostrar que los programas de ajustes estructurales afectan negativamente estos derechos. ${ }^{9}$

En su informe como experto independiente para el Consejo de Derechos Humanos de las Naciones Unidas, Bohoslavsky resalta de manera contundente los shocks adversos que los programas del FMI ocasionan sobre el trabajo en varios países en vías de desarrollo. ${ }^{10}$ Expone que las reformas relacionadas con el trabajo impulsadas por medidas de austeridad han resultado en la desregulación del derecho laboral. Concretamente, la condicionalidad laboral, que permanece como una característica importante de los programas de asistencia financiera del FMI, socava los sistemas de negociación colectiva y los sindicatos, al mismo tiempo que incrementa los conflictos políticos y la inseguridad económica de los países prestatarios.

La mayor parte de los estudios analizados anteriormente estima los efectos de los programas del FMI a través de la comparación del estado anterior y posterior a la participación en dichos programas o a través de la comparación del estado con o sin participación en los programas. Estudios más recientes tienden a ser más elaborados en cuanto a la metodología. Con frecuencia, toman en consideración los efectos de selección antes de evaluar el efecto de la participación en programas del FMI. No obstante, al establecer como el principal factor explicativo una variable dicotómica de la participación en programas del FMI, estos estudios asumen de manera implícita que todos los programas del FMI tienen un diseño similar o que dichos

9 Robert G. Blanton, Shannon Lindsey Blanton y Dursun Peksen, "The Impact of IMF and World Bank Programs on Labor Rights", 2 Political Research Quarterly, 2015, Vol. 68, 324-336.

10 Juan P. Bohoslavsky, "Report of the Independent Expert on the Effects of Foreign Debt and Other Related International Financial Obligations of States on the Full Enjoyment of All Human Rights, Particularly Economic, Social and Cultural Rights", United Nations Human Rights Council, A/HRC/34/57, 2017. 
programas tendrían efectos parecidos una vez firmados, sin importar su condicionalidad. Muchos estudios recientes demuestran que existen grandes variaciones en los diseños de los programas del FMI, como por ejemplo la cantidad de condiciones incluidas, ${ }^{11}$ el alcance de las condicionalidades comprendidas en el programa ${ }^{12}$ y las condicionalidades relativas al trabajo. ${ }^{13}$

La falta de atención a la variación de las condicionalidades aparenta ser problemática, dado que el razonamiento teórico detrás de la conexión entre los programas del FMI y los derechos laborales resalta el rol central de aquellas. Los estudios analizados anteriormente que examinan el efecto de los programas del FMI con frecuencia explicitan que las condicionalidades comprendidas en dichos programas obligan a los gobiernos a adoptar políticas que comprometen los derechos de los/as trabajadores/as significativamente, como la restricción al aumento de salarios en el sector público, que apunta directamente a los/as trabajadores/as, o la disminución del gasto social, que afecta a los/as trabajadores/as de manera indirecta. Sin embargo, en los análisis empíricos de estos estudios, no se pone atención a la variación de condiciones del mercado laboral específicas que se incluyen en los programas del FMI. Debido a las exigencias de intereses locales y a la variada capacidad de reacción de los gobiernos, algunos programas tienen pocas condiciones sobre el mercado laboral, o ninguna, mientras que otras incluyen una cantidad considerable de condiciones. ${ }^{14}$ Con

\footnotetext{
11 Axel Dreher y Nathan Jensen, "Independent Actor or Agent? An Empirical Analysis of the Impact of US Interests on IMF Conditions", The Journal of Law and Economics, 2007, Vol. 50:1, 105-124; Mark Copelovitch, "Master or Servant? Agency Slack and the Politics of IMF Lending", 1 International Studies Quarterly, 2010, Vol. 54, 49-77; Byungwon Woo, The Strategic Politics of IMF Conditionality, (Tesis de doctorado, Universidad de Ohio, 2010).

12 Randall W. Stone, "The Scope of IMF Conditionality", 4 International Organization, 2008, Vol. 62, 589-620.

13 Teri Caraway, Stephanie Rickard y Mark Anner, "International Negotiations and Domestic Politics: The Case of IMF Labor Market Conditionality", 1 International Organization, 2012, Vol. 66, 27-61.
}

14 Ibid. 
base en los últimos estudios sobre las consecuencias de los programas del FMI, consideramos que es poco razonable esperar que estos programas diversos tengan efectos similares en los derechos laborales. ${ }^{15}$ Nuestra intención es abordar este tema a través del análisis de los efectos que las condicionalidades del FMI, específicamente aquellas relativas al mercado laboral, tiene sobre los derechos de los/as trabajadores/as.

\section{Argumento teórico}

Los programas del FMI se negocian, conforme lo acordado internacionalmente, entre el FMI y cada gobierno. Una vez logrado el acuerdo, el programa se implementa a nivel local. Desde un punto de vista analítico, estos dos procesos secuenciales se encuentran íntimamente relacionados. Asimismo, en el marco de los préstamos otorgados por el FMI, puede entenderse que el diseño de un programa del FMI y las políticas nacionales de implementación de políticas de reformas, tanto como los efectos producidos por estas, también están vinculados estrechamente. El diseño de un programa del FMI debería contemplar la viabilidad de su implementación. En particular, al momento de diseñarse los programas del FMI, un punto clave que debería contemplarse a nivel político es la posibilidad de los/as trabajadores/as y los sindicatos de resistir las reformas del mercado laboral. Debido a esto, tanto la probabilidad de lograr una implementación exitosa como la lucha política a nivel local sobre las reformas del mercado laboral dependen de la forma en que se diseñe el programa del FMI. Por lo tanto, desde el plano conceptual, concebimos la negociación de los programas del FMI y su implementación como un proceso de dos etapas, similar a la conceptualización de los juegos en dos

15 Quintin Beazer y Byungwon Woo, "IMF Conditionality, Government Partisanship, and the Progress of Economic Reforms", 2 American Journal of Political Science, 2016, Vol. 60, 304321; Byungwon Woo, "Conditional on Conditionality: IMF Program Design and Foreign Direct Investment", 3 International Interactions, 2013, Vol. 39, 292-315. 
niveles, es decir: por un lado, la negociación del programa del FMI, que se lleva a cabo entre el FMI y un gobierno nacional; y, por otro lado, las políticas de implementación del programa del FMI, en pugna entre el gobierno nacional y la posible oposición de los/as trabajadores/as y los sindicatos una vez acordado el programa. Y, de forma intrínseca, la interacción entre el diseño del programa y su implementación es lo que determina el resultado: los derechos de los/as trabajadores/as.

Si bien las políticas nacionales de implementación de un programa imponen límites al FMI y al gobierno nacional, sería erróneo afirmar que las limitaciones nacionales son determinantes para definir la condicionalidad del mercado laboral en un programa del FMI. Mientras que las limitaciones a nivel local afectan, de forma evidente y hasta cierto punto, el diseño de un programa del FMI, tanto los intereses estratégicos y burocráticos del FMI como las motivaciones políticas del gobierno involucrado entran en juego en gran medida. Esto se debe a la incertidumbre que se genera alrededor de la manera en que las políticas de implementación de las medidas de reforma del mercado laboral pueden impactar a futuro. Asimismo, la oposición laboral es, en parte, endógena a la forma en que se diseña un programa del FMI. Por lo tanto, un gobierno puede intentar usar los programas del FMI con objetivos políticos. Por ejemplo, estudios existentes del FMI han demostrado que un gobierno puede implementar un programa del FMI con el propósito de inclinar la balanza en contra de los grupos opositores a las reformas, entre los que se incluyen, probablemente, los sindicatos. ${ }^{16}$ Mediante la incorporación del FMI al debate, un gobierno que busca implementar reformas puede lograr que el costo de rechazar las iniciativas de esta índole sea mayor para los sindicatos. De igual manera, otros han demostrado que los intereses estratégicos estadounidenses y los intereses burocráticos y organizacionales propios de los empleados del

16 James Raymond Vreeland, The IMF and Economic Development, (Cambridge University Press, Cambridge, 2003). 
FMI influyen sobre varios aspectos de los programas del FMI. En otras palabras, al momento de negociar un programa del FMI, tanto este como el gobierno involucrado gozan de una autonomía limitada.

Los resultados de esta negociación, es decir, las condicionalidades, varían significativamente. Estudios recientes sobre las condicionalidades del FMI muestran que existen variaciones notables en la cantidad total o en su alcance. Asimismo, el estudio de las condicionalidades del FMI sobre el mercado laboral comprueba que las condiciones en las reformas del mercado laboral incluidas en los programas del FMI difieren considerablemente. ${ }^{17}$ De todos los programas celebrados entre los años 1980 y 2000, la cantidad promedio de condiciones relativas al trabajo en los programas del FMI asciende a 1.69 según las cartas acuerdo o a 5.26 según las cartas de intención, con una desviación estándar de 3.43 y 5.13, respectivamente. La diferencia entre las cantidades mínimas y máximas resulta aún más sorprendente. Mientras que varios programas no establecen condiciones relacionadas con el trabajo, otros pueden incluir 20, 30 o incluso 40 condiciones.

Existen estudios que no le dan importancia a esta variación. Los académicos que seleccionan la participación en los programas del FMI (una variable dicotómica) como el principal factor explicativo imponen el supuesto implícito de que todos los programas del FMI están diseñados de forma similar o producen efectos homogéneos sin importar su diseño. No obstante, observamos que los programas del FMI pueden ser muy distintos unos de otros. ${ }^{18}$ Es poco probable que programas tan heterogéneos afecten a los países involucrados de manera

\footnotetext{
17 Teri Caraway, Stephanie Rickard y Mark Anner, "International Negotiations and Domestic Politics: The Case of IMF Labor Market Conditionality".

18 Axel Dreher y Nathan Jensen, "Independent Actor or Agent? An Empirical Analysis of the Impact of US Interests on IMF Conditions"; Randall W. Stone, "The Scope of IMF Conditionality"; Mark Copelovitch, "Master or Servant? Agency Slack and the Politics of IMF Lending"; Byungwon Woo, The Strategic Politics of IMF Conditionality.
} 
uniforme. Por lo tanto, suponemos que el enfoque empírico convencional de la bibliografía existente oculta facetas importantes de los efectos de los programas del FMI en los derechos de los/as trabajadores/as.

Desde nuestra perspectiva, sostenemos que cuantas más condiciones relativas al trabajo se incluyan en un programa, más negativo será el impacto en los derechos de los/as trabajadores/as durante el año siguiente. En otras palabras, cuando un programa incluye una gran cantidad de condiciones relativas al mercado laboral, se realizan mayores concesiones posteriores en materia de derechos de los/as trabajadores/as que cuando no las incluye. Las condiciones en torno al trabajo comprenden restricciones en los niveles de los salarios y los niveles de empleo en el sector público, la privatización, los salarios mínimos en el sector privado, otras restricciones salariales en el sector privado, la seguridad social, las pensiones públicas, la flexibilidad en el mercado laboral y la descentralización de la negociación colectiva. ${ }^{19}$ Estas condiciones explícitas relativas al mercado laboral afectan los intereses de los/as trabajadores/as. Debido a esto, y a que mantienen otras variables constantes, a medida que las condicionalidades laborales se tornan más estrictas, los derechos de los/as trabajadores/as se deterioran más.

Hipótesis 1a: Cuando un programa del FMI incluye condiciones del mercado laboral más estrictas, es más probable que el programa afecte negativamente a los derechos de iure de los/as trabajadores/as.

Hipótesis 1b: Cuando un programa del FMI incluye condiciones del mercado laboral más estrictas, es más probable que el programa afecte negativamente a las prácticas laborales de facto.

19 Teri Caraway, Stephanie Rickard y Mark Anner, "International Negotiations and Domestic Politics: The Case of IMF Labor Market Conditionality". 
Asimismo, sostenemos que existen algunos factores políticos a nivel local que pueden intervenir y mitigar los efectos negativos de las condiciones sobre el mercado laboral. Cabe destacar que, inicialmente, señalamos que no se implementa la totalidad de las condicionalidades relativas al trabajo. De hecho, la implementación de las condiciones del FMI no se da por garantizada debido a que, con frecuencia, se enfrenta a una firme resistencia a nivel local. Y tanto el FMI como el gobierno local tienen intenciones de hacer concesiones cuando se encuentran ante estas situaciones. Algunos programas se descartan por completo y vuelven a negociarse. Otros, en cambio, avanzan con leves modificaciones de las condiciones. En otras instancias, el incumplimiento de ciertas condiciones no siempre se ve sancionado por el FMI, pero, a cambio, se otorgan ciertas exenciones. Sostenemos que, cuando los/as trabajadores/as tienen mayor representación política o más canales de influencia, es posible mitigar el efecto negativo de la implementación de las condiciones del mercado laboral. Desde nuestra perspectiva, uno de dichos casos tiene lugar cuando el gobierno participante es de izquierda. Cuando una fuerza de izquierda tiene mejor representación política en un país, es posible lograr concesiones respecto de la implementación de las condiciones sobre el mercado laboral, o bien, pueden modificarse, lo que contrarresta parcialmente el efecto negativo de las condiciones sobre el mercado laboral en los derechos de los/as trabajadores/as.

Hipótesis 2a: Cuando un gobierno de izquierda participa en un programa del FMI que incluye condiciones del mercado laboral más estrictas, es menos probable que el programa afecte negativamente a los derechos de iure de los/as trabajadores/as que cuando un gobierno de derecha participa de dicho programa.

Hipótesis 2b: Cuando un gobierno de izquierda participa en un programa del FMI que incluye condiciones del mercado laboral más estrictas, es menos probable que el programa afecte negativamente a las prácticas laborales de 
facto que cuando un gobierno de derecha participa de dicho programa.

Existe una vasta bibliografía en cuestiones de economía política comparativa que demuestra que las características institucionales de los sistemas políticos locales están asociadas significativamente con el desarrollo de las políticas económicas y los resultados de las políticas públicas. Las instituciones políticas afectan la motivación y la capacidad de los gobiernos para responder a las demandas colectivas de intereses locales, ya que estos definen las formas en que los representantes políticos maximizan sus oportunidades para conservar el poder. ${ }^{20}$ Son varias las instituciones políticas a nivel local, entre ellas, los tipos de régimen, las fórmulas electorales y los sistemas partidarios, las que influyen en la voluntad de los gobiernos para integrarse en la economía internacional ${ }^{21}$ y para interceder ante el efecto de los cambios externos y los impactos en los resultados de las políticas a nivel local. ${ }^{22}$

Otra repercusión importante que surgió de estos estudios es que la relación entre las condiciones de los programas del FMI que se relacionan con el trabajo y la protección nacional de iure y de facto de los derechos laborales podría verse condicionada

20 Gian Maria Milesi-Ferretti, Roberto Perotti y Massimo Rostagno, "Electoral Systems and Public Spending", 2 The Quarterly Journal of Economics, 2002, Vol. 117, 609-657; Allen Hicken y Joel W. Simmons, "The Personal Vote and the Efficacy of Education Spending", 1 American Journal of Political Science, 2008, Vol. 52, 109-124; Stephanie J. Rickard, Spending to Win: Political Institutions, Economic Geography, and Government Subsidies, (Cambridge University Press, Cambridge, 2018).

21 Helen V. Milner y Bumba Mukherjee, "Democratization and Economic Globalization", Annual Review of Political Science, 2009, Vol. 12, 163-181; Sean D. Ehrlich, "Access to Protection: Domestic Institutions and Trade Policy in Democracies", 3 International Organization, 2007, Vol. 61, 571-605.

22 Alícia Adserà y Carles Boix, "Trade, Democracy, and the Size of the Public Sector: The Political Underpinnings of Openness", 2 International Organization, 2002, Vol. 56, 229-262; Robert Kaufman y Alex Segura-Ubiergo, “Globalization, Domestic Politics, and Social Spending in Latin America 1973-1997: A Cross-Sectional Time Series Analysis", 4 World Politics, 2001, Vol. 53, 553587. 
por las instituciones políticas locales. Incluso si se proponen estrictas reformas al mercado laboral en la instancia de negociaciones y acuerdos para obtener préstamos del FMI, sus efectos en los derechos colectivos del trabajo pueden variar en función de la medida en que las instituciones políticas garanticen la representación política de los/as trabajadores/as y los sindicatos.

En este punto, sostenemos que los sistemas electorales condicionan la relación entre la condicionalidad del mercado laboral y los derechos colectivos del trabajo. Las investigaciones existentes parecen indicar que hay una fuerte afinidad entre los sistemas de representación proporcional (en adelante, RP) y las políticas de clase de amplio alcance. A diferencia de los sistemas mayoritarios con distritos uninominales, los sistemas de RP están fuertemente asociados con un gasto público elevado, baja igualdad y mayor redistribución. ${ }^{23}$ La baja elasticidad en la relación de escaños respecto a votos en los sistemas de RP tiende a generar un sistema multipartidista, lo que facilita en gran medida el ingreso de nuevos partidos que representen los intereses de los/as trabajadores/as y los sindicatos. Hay más posibilidades de que los sistemas de RP cumplan con los beneficios colectivos del trabajo local que los sistemas mayoritarios, debido a que los partidos de coalición de los sistemas de RP tienen una tendencia menor a desviarse de la preferencia de los principales seguidores del partido. ${ }^{24}$ En particular, un sistema de RP de lista cerrada en el que los rótulos partidarios importan más que las reputaciones personales permite que la fuerza laboral organizada ejerza y sancione a los/as representantes electos mediante los partidos políticos. ${ }^{25}$

23 Birchfield, Vicki y Crepaz, Markus M. L. "The Impact of Constitutional Structures and Collective and Competitive Veto Points on Income Inequality in Industrialized Democracies", 2 European Journal of Political Research, 1998, Vol. 34, 175-200; Iversen, Torben y Soskice, David. "Electoral Institutions and the Politics of Coalitions: Why Some Democracies Redistribute More than Others", 2 American Political Science Review, 2006, Vol. 100, 165-181.

24 Ibid.

25 Alberto Alesina y Edward Glaeser, Fighting Poverty in the US and Europe: A World of Difference, (Oxford University Press, 2004). 
En esta línea, nuestra hipótesis es que los sistemas de RP de lista cerrada o los altos niveles de proporcionalidad de los sistemas electorales anularían los efectos negativos de las estrictas condiciones laborales sobre los derechos colectivos del trabajo. Las magnitudes de distrito grandes en los sistemas de RP de lista cerrada aumentarían las posibilidades de que las exigencias de la fuerza laboral organizada para proteger los derechos laborales se traduzcan en votos reales y, en consecuencia, inducirían a los legisladores representantes a proteger los derechos laborales de iure y de facto.

Hipótesis 3a: Cuando un país que adopta un sistema de RP de lista cerrada participa en un programa del FMI que incluye condiciones del mercado laboral más estrictas, es menos probable que el programa afecte negativamente a los derechos de iure de los/as trabajadores/as que cuando un país con otros tipos de sistemas electorales (como por ejemplo, sistemas mayoritarios, semiproporcionales o de RP de lista abierta) participa en dicho programa.

Hipótesis 3b: Cuando un país que adopta un sistema de RP de lista cerrada participa en un programa del FMI que incluye condiciones del mercado laboral más estrictas, es menos probable que el programa afecte negativamente a los derechos de facto de los trabajadores que cuando un país con otros tipos de sistemas electorales (como por ejemplo, sistemas mayoritarios, semiproporcionales o de RP de lista abierta) participa en dicho programa.

En síntesis, nuestra teoría es que el contenido de un programa del FMI determina de forma crucial el efecto del programa en los derechos de los/as trabajadores/as. Asimismo, las circunstancias políticas locales pueden intervenir en el proceso y mitigar el efecto global. 


\section{Datos y métodos}

En primer lugar, explicamos las principales variables dependientes e independientes de nuestro modelo. Luego, discutimos la aplicación de conditional mixed-process models (modelos CMP, por sus siglas en inglés) con el objetivo de evitar el sesgo en la selección dentro del análisis del impacto de los programas del FMI en los derechos laborales.

\section{Variables dependientes}

Nuestras variables dependientes son los indicadores, propuestos por Mosley y Uno, de los derechos colectivos del trabajo de 90 países en vías de desarrollo entre 1985 y 2002. ${ }^{26}$ Sobre la base de la plantilla de estándares laborales de 37 categorías, propuesta por Kucera, ${ }^{27}$ Mosley y Uno construyen un conjunto de datos internacionales sobre violaciones a los derechos laborales dentro de las siguientes seis áreas: derecho de asociación, derecho a formar y a unirse a sindicatos, derecho a realizar otras actividades sindicales, derecho a entablar negociaciones colectivas, derecho de huelga y derechos en zonas francas industriales (ZFI). Su evaluación de los estándares laborales se funda en el análisis del contenido de observaciones provenientes de tres fuentes: el Departamento de Estado de los EE. UU., la Organización Internacional del Trabajo y la Confederación Internacional de Organizaciones Sindicales Libres (CIOSL). Mosley y Uno calculan la suma ponderada de violaciones a los derechos laborales en las 37 categorías antes mencionadas, por país y por año, otorgándole mayor ponderación a las violaciones más graves. Posteriormente, al revertir la escala de las violaciones a los

26 Layna Mosley y Saika Uno, "Racing to the Bottom or Climbing to the Top? Economic Globalization and Collective Labor Rights",8 Comparative Political Studies, 2007, Vol. 40, 923-948.

27 David Kucera, "Core Labour Standards and Foreign Direct Investment", 1/2 International Labor Review, 2002, Vol. 141, 31-69. 
derechos laborales, generan puntajes que indican la medida de respeto por los derechos colectivos del trabajo. Cuanto mayor es el valor del puntaje, mayor respeto, y viceversa.

Con las mediciones anuales de violaciones a los derechos laborales tomadas por Mosley y Uno, Greenhill, Mosley y Prakash crean otras dos variables que comprenden aspectos de iure y de facto de los derechos colectivos del trabajo, respectivamente. ${ }^{28}$ Labor Laws (Leyes laborales) representa el alcance de la legislación local en lo que respecta a la definición y el reconocimiento de los derechos de los/as trabajadores/as a organizarse, a negociar de manera colectiva y a hacer huelgas. Labor Practices (Prácticas laborales) indica el alcance de la violación y limitación de dichos derechos laborales por parte de funcionarios del gobierno o de empleadores de los sectores públicos y privados. Labor Laws y Labor Practices equivalen a la suma ponderada de las violaciones a los elementos de la ley y la práctica expuestos por Kucera en sus 37 categorías de estándares laborales. La diferencia entre las leyes y las prácti cas sugiere que la legislación formal sobre los derechos de los/ as trabajadores/as no garantiza necesariamente el ejercicio real de dichos derechos, en especial si los gobiernos carecen de habilidad para controlar y hacer valer los estándares laborales en la práctica. Por lo tanto, nuestro análisis se concentra principalmente en los efectos de las condiciones del FMI en las leyes y las prácticas laborales. También consideramos los puntajes de derechos laborales de Mosley y Uno. ${ }^{29}$

\section{Variables independientes}

Nuestras variables independientes clave son las mediciones de la condicionalidad del mercado laboral del FMI tomadas

\footnotetext{
28 Brian Greenhill, Layna Mosley y Aseem Prakash, “Trade-based Diffusion of Labor Rights: A Panel Study, 1986-2002",4 American Political Science Review, 2009, Vol. 103, 669-690.

29 Layna Mosley y Saika Uno, "Racing to the Bottom or Climbing to the Top? Economic Globalization and Collective Labor Rights".
} 
por Caraway, Rickard y Anner. ${ }^{30}$ Estas mediciones cuantifican la severidad de las condiciones de las reformas del mercado laboral para recibir préstamos del FMI a partir de los dos tipos de documentos oficiales del FMI. Las Cartas de intención presentan las evaluaciones de los problemas más graves de las economías de los países prestatarios y su disposición a promulgar programas de reformas para solucionar los problemas antes mencionados. Las Cartas acuerdo cumplen la función de contrato de préstamo, ya que detallan las condiciones que el FMI exige cumplir a los países prestatarios para ser receptores de los préstamos de la institución. A raíz de las potenciales diferencias entre las condiciones relacionadas con el trabajo que se proponen en estos dos documentos, medimos los efectos de la severidad de las condiciones laborales propuestas en Letters of Intent y en Arrangement Letters sobre aspectos de iure y de facto de los derechos colectivos del trabajo, respectivamente. Los valores positivos altos en Letters of Intent y en Arrangement Letters dan cuenta de una mayor intrusión de la condicionalidad del mercado laboral. En el caso de que dicha condicionalidad afecte de manera adversa los derechos colectivos del trabajo, los coeficientes de Letters of Intent y de Arrangement Letters deberían ser negativos y significativos a nivel estadístico.

Nuestras variables independientes también incluyen los sistemas electorales y la inclinación ideológica de los gobiernos. PR (Representación Proporcional) es una variable ficticia que asigna el valor 1 a los sistemas de RP de lista partidaria en los que, al menos en uno de los niveles electorales, los líderes del partido controlan el orden de los/as candidatos/as en las votaciones y los electores votan por el partido, no por candidatos/as individuales. Left (izquierda) es una variable ficticia que recibe el código 1 en el caso de que el partido del Ejecutivo sea de izquierda o centrista. De lo contrario, recibe el código 0.

\footnotetext{
30 Teri Caraway, Stephanie Rickard y Mark Anner, "International Negotiations and Domestic Politics: The Case of IMF Labor Market Conditionality".
} 
Asimismo, utilizamos un conjunto de variables de control que pueden afectar los niveles de respeto de los derechos laborales. En línea con la bibliografía sobre los efectos de la globalización en los derechos laborales, ${ }^{31}$ consideramos tres variables diferentes que registran el grado de integración de un país en la economía mundial. Trade (comercio) señala la suma de las importaciones y exportaciones como parte del PBI y FDI (Inversión Extranjera Directa, IED) indica la cantidad de ingresos por inversiones extranjeras directas como porcentaje de participación en el PBI. Con el objetivo de controlar el efecto del comercio en la transmisión de derechos laborales a través de las fronteras, utilizamos las mediciones de Bilateral Trade Context (Contexto de Comercio Bilateral) de Greenhill et al., que indican el nivel agregado de los estándares laborales de los socios exportadores de un país. ${ }^{32}$ Aquel estudio genera la variable Bilateral Trade Context para Labor Laws y Labor Practices por país y año, respectivamente, por medio del cálculo de cada suma ponderada de los puntajes de las leyes y las prácticas de los socios exportadores de un país, ponderando la parte del socio exportador en el total de exportaciones de un país. En el caso de que los derechos colectivos del trabajo se vean afectados por los estándares laborales de sus destinos de exportación, los coeficientes de las mediciones de Bilateral Trade Context serán positivos y significativos. ${ }^{33}$

Con el objeto de mantener constantes los efectos de Democracy (Democracia), utilizamos los puntajes Polity del Proyecto Polity IV, que varían desde el -10 para identificar autocracias plenas hasta el 10 para identificar democracias plenas. ${ }^{34}$ Conflict

31 Layna Mosley y Saika Uno, "Racing to the Bottom or Climbing to the Top? Economic Globalization and Collective Labor Rights".

32 Brian Greenhill, Layna Mosley y Aseem Prakash, "Trade-based Diffusion of Labor Rights: A Panel Study, 1986-2002".

33 Ibid. Greenhill et al. proponen mediciones de Bilateral Trade Context únicamente para Labor Laws y Labor Practices. Por lo tanto, usamos el valor promedio cuando el modelo se evalúa con los puntajes de derechos laborales de Mosley y Uno.

34 Monty G.Marshall, Keith Jaggers y Ted Robert Gurr, "Polity IV Project: Political Regime Characteristics and Transitions, 1800-2011", Center for Systemic Peace, 2011. 
(Conflicto) es una variable ficticia que hace referencia a conflictos armados, tanto internos como interestatales. Para determinarla, se utilizó el conjunto de datos UCDP/PRIO Armed Conflict Dataset. ${ }^{35}$ Nuestros modelos también incluyen el tamaño de la población y el PBI per cápita, todo medido en logaritmos.

\section{Métodos de estimación}

Los estudios existentes sugieren que el problema de selección debe tratarse cuidadosamente a la hora de analizar los efectos de los programas del FMI. ${ }^{36}$ La participación de un país en un programa del FMI no es una decisión azarosa, ya que es probable que los países con resultados económicos desfavorables soliciten préstamos del FMI en momentos de crisis económicas. Dicha endogeneidad en las variables relacionadas con el FMI sesgaría nuestras estimaciones, en especial si un conjunto de factores asociados a la participación en programas del FMI y a las condiciones de los préstamos también afectara los niveles de derechos colectivos del trabajo en los países prestatarios.

Con el objeto de abordar estas cuestiones, utilizamos el método conditional mixed-process (CMP) propuesto por Roodman. ${ }^{37}$ Este método se usa con frecuencia en los estudios existentes que analizan los determinantes externos de los derechos laborales. ${ }^{38}$

35 Nils Petter Gleditsch, Peter Wallensteen, Mikael Eriksson, Margareta Sollenberg y Håvard Strand, "Armed Conflict 1946-2001: A New Dataset", 5 Journal of Peace Research, 2002, Vol. 39, 615-637.

36 James Raymond Vreeland, The IMF and Economic Development; Axel Dreher, "IMF and Economic Growth: The Effects of Programs, Loans, And Compliance with Conditionality", 5 World Development, 2006, Vol. 34, 769-788; Rodwan Abouharb y David L. Cingranelli, "IMF Programs and Human Rights, 1981-2003", 1 The Review of International Organizations, 2009, Vol. 4, 47-72.

37 David Roodman, "Fitting Fully Observed Recursive Mixed-Process Models with CMP", 2 Stata Journal, 2011, Vol. 11, 159-206.

38 Robert G. Blanton, Shannon Lindsey Blanton y Dursun Peksen, "The Impact of IMF and World Bank Programs on Labor Rights"; Nicole Detraz y Dursun Peksen, "The Effect of IMF 
Al usar una técnica de regresión aparentemente no relacionada (SUR, del inglés Seemingly Unrelated Regression), el método CMP nos permite estimar de manera simultánea los determinantes de los derechos colectivos del trabajo y las condiciones del FMI sobre el mercado laboral. El modelo CMP consiste de dos ecuaciones con errores correlacionados. La primera ecuación utiliza como variables dependientes cada uno de los indicadores de condicionalidad laboral del FMI (es decir, Letters of Intent y Arrangement Letters) y considera un conjunto de factores político-económicos que podrían afectar la severidad de la condicionalidad laboral. La segunda ecuación analiza los efectos de la condicionalidad del FMI y su interacción con los sistemas electorales y la inclinación ideológica de los gobiernos en cuanto a los derechos colectivos del trabajo (es decir, Labor Laws y Labor Practices), junto con las variables de control que se explican anteriormente.

Varios estudios sugieren que hay una relación sistemática entre las condicionalidades del FMI y la relevancia política del país prestatario en la política internacional. Los países que se encuentran estrechamente alineados con los intereses estratégicos de los Estados Unidos tienden a recibir préstamos del FMI que establecen menor cantidad de condiciones ${ }^{39}$ o préstamos más cuantiosos. ${ }^{40}$ De manera similar, los países que son miembros temporarios del Consejo de Seguridad de las Naciones Unidas (UNSC, por sus siglas en inglés) reciben un trato favorable, con condiciones menos estrictas en los préstamos del FMI, debido a que la mayoría de los accionistas del FMI buscan

Programs on Women's Economic and Political Rights", 1 International Interactions, 2016, Vol. 42, 81-105; Dursun Peksen y Robert G. Blanton, "The Impact of ILO Conventions on Worker Rights: Are Empty Promises Worse than No Promises?", 1 The Review of International Organizations, 2017, Vol. 12, 75-94.

39 Axel Dreher y Nathan Jensen, "Independent Actor or Agent? An Empirical Analysis of the Impact of US Interests on IMF Conditions".

40 Thomas Oatley y Jason Yackee, "American interests and IMF lending", 3 International, 2004, Vol. 41, 415-429. 
obtener influencia política en el Consejo de Seguridad. ${ }^{41}$ Para controlar dichos efectos geopolíticos, en la primera ecuación se utilizan dos variables. UNGA Voting (Votación UNGA) es una variable recopilada de Bailey et al. que indica la similitud de votos entre un país y los Estados Unidos en la Asamblea General de las Naciones Unidas (UNGA, por sus siglas en inglés). ${ }^{42}$ Dentro de las tres opciones de voto (es decir, afirmativo, negativo y abstención), la similitud de votación se mide como el número total de votos sobre un determinado asunto en el que el país en cuestión y los Estados Unidos coincidieron, dividido por el total de votos de ambos países. Una variable ficticia que indica la membresía temporal de un país en el UNSC considera los efectos de las consideraciones geopolíticas sobre las condiciones de los préstamos del FMI.

La primera ecuación sobre la condicionalidad del FMI incluye distintas covariables. Los efectos de Democracy nuevamente se mantienen constantes a través de los puntajes Polity del Proyecto Polity IV. Left Party Power (Poder del partido de izquierda), que captura la representación política de partidos de izquierda, refleja el valor promedio entre las orientaciones partidarias del Ejecutivo, el partido oficialista más grande y el partido opositor más grande. Derecha, centro e izquierda están codificados como 1, 2 y 3, respectivamente. ${ }^{43}$ Exchange Rate (Tasa de cambio) indica el monto de la moneda local equivalente a un dólar estadounidense. ${ }^{44}$ Tanto GDP per cápita (PBI per cápita) como GDP (PBI) y Total Reserves (total de reservas),

41 Axel Dreher, Jan-Egbert Sturm y James Raymond Vreeland, "Politics and IMF Conditionality", 1 Journal of Conflict Resolution, 2015, Vol. 59, 120-148.

42 Michael Bailey, Anton Strezhnev y Erik Voeten, “Estimating Dynamic State Preferences from United Nations Voting Data", 2 Journal of Conflict Resolution, 2017, Vol. 61, 430-456.

43 Daniel Berliner, Anne Greenleaf, Milli Lake y Jennifer Noveck, "Building Capacity, Building Rights? State Capacity and Labor Rights in Developing Countries", World Development, 2015, Vol. 72, 127-139.

44 El valor de Exchange Rate se divide por 100 en todas las tablas de regresión para una mejor presentación. 
expresadas en miles de millones de dólares estadounidenses, se miden en logaritmos y se las incluye como factores que podrían afectar las condicionalidades del FMI. Todas las estimaciones de la primera ecuación incluyen una tendencia temporal, ya que la cantidad y el alcance de las condiciones de los préstamos del FMI han aumentado a través de los años. ${ }^{45}$

Los datos sobre sistemas electorales y partidismo fueron obtenidos de la versión de 2012 de la base de datos de instituciones políticas (DPI). ${ }^{46}$ Los datos de Exchange Rate fueron tomados de la División de Estadísticas de las Naciones Unidas, mientras que los datos del resto de las variables socioeconómicas provienen de los Indicadores de Desarrollo Mundial del Banco Mundial (del inglés World Bank's World Development Indicators) del año 2013.

\section{Resultados empíricos}

En esta sección, presentamos los resultados obtenidos respecto del impacto de las condiciones de los préstamos del FMI y los derechos colectivos del trabajo. Nuestro enfoque se centra en el análisis de dos temas: en primer lugar, si las condiciones relacionadas con el trabajo que se incluyen en los documentos sobre préstamos del FMI afectan los derechos laborales en los países prestatarios y, en caso afirmativo, hasta qué punto; y en segundo lugar, en qué medida los factores políticos locales, como los sistemas electorales y el partidismo de los gobiernos, condicionan la relación sugerida entre la condicionalidad del FMI y los derechos laborales.

En la tabla 1 se presentan los efectos de Letters of Intent en los derechos laborales de jure y de facto. La tabla 1 cuenta con

\footnotetext{
45 Teri Caraway, Stephanie Rickard y Mark Anner, "International Negotiations and Domestic Politics: The Case of IMF Labor Market Conditionality".

46 Philip Keefer, "Database of Political Institutions 2012", World Bank Working Paper Series, 2012, No. 2283.
} 
dos partes, ya que analizamos, simultáneamente, los determinantes de los derechos colectivos del trabajo y la condicionalidad del FMI con un proceso de estimación CMP. En la parte superior, se muestra el impacto de la condicionalidad laboral del FMI recopilada por Letters of Intent, en Labor Laws y Labor Practices, y se mantiene constante el resto de las covariables mencionadas en la sección anterior. En la parte inferior, se presenta un conjunto de factores que están asociados a la severidad de las condiciones en torno al trabajo incluidas en Letters of Intent. ${ }^{47}$

Los resultados de la tabla 1 parecen indicar que la intrusión de las condicionalidades del FMI en Letters of Intent tiende a socavar el nivel de respeto de un país por las leyes y las prácticas laborales. Las estimaciones de referencia en los modelos 1 y 6 indican que la asociación negativa de Letters of Intent con las leyes y las prácticas solo resulta significativa para estas últimas. Sin embargo, una vez que analizamos la interacción de Letters of Intent con el partidismo de los gobiernos o los sistemas electorales, observamos que los coeficientes para Letters of Intent son negativos y significativos en forma casi constante.

Los efectos sustantivos de Letters of Intent deberían interpretarse con cautela si el modelo incluye términos de interacción multiplicadores entre Letters of Intent y otras variables. Por ejemplo, los resultados del modelo 3 parecen indicar que un aumento de una unidad en Letters of Intent disminuiría el nivel

\footnotetext{
47 En pocas palabras, en la tabla 1 se muestran los resultados clave de nuestro análisis. La parte superior no muestra los términos constantes, los valores ficticios regionales ni los coeficientes para los valores controlados de la ecuación de los derechos laborales (por ejemplo, Democracy (Democracia), Conflict (Conflicto), Trade (Comercio), FDI (IED), Log population (Log población), Log per capita GDP (Log PBI per cápita), Bilateral Trade Context (Contexto de comercio bilateral) y Lagged Dependent Variable (Variable dependiente rezagada). Asimismo, la parte inferior omite los términos constantes y los coeficientes para los valores controlados de la ecuación de la condicionalidad laboral del FMI (por ejemplo, Democracy, UNSC y UNGA Voting). Dicho formato se usa en las tablas 2 y 5 . En las tablas restantes se presentan nuestros hallazgos más importantes. Los resultados completos se encuentran disponibles a pedido.
} 
de Labor Laws en 0.337 puntos si los países no adoptan RP de lista cerrada. No obstante, el coeficiente Letters of Intent $\times P R$ $(=0.177)$ indica que el mismo cambio en Letters of Intent generaría una disminución de Labor Laws en 0.16 puntos en países con RP de lista cerrada. Los resultados se mantienen práctica y sustancialmente iguales cuando aplicamos el modelo con las submuestras de información. En los modelos 4 y 5 queda demostrado que un escenario con RP de lista cerrada cambia el efecto marginal de Letters of Intent sobre Labor Laws de -0.663 a -0.498 en democracias (con puntajes Polity $>0$ ) y de -1.243 a -0.523 en democracias estables (con puntajes Polity $>5$ ). De forma similar, en los modelos 8,9 y 10, los coeficientes positivos y significativos de la ecuación Letter of Intent $\times P R$ indican que la medida en la que las condicionalidades del FMI afectan Labor Practices negativamente se mitigará en los sistemas con RP de lista cerrada, en especial, en casos de monarquías estables y consolidadas.

Los efectos del partidismo de los gobiernos (=Left) en la relación entre las condicionalidades del FMI y los derechos laborales no están tan claros como los efectos de la RP de lista cerrada. La estimación de coeficientes de Letter of Intent $\times$ Left resulta positiva para Laws en el modelo 2, pero negativa para Practices en el modelo 7. Particularmente, en el modelo 7 se refleja que el efecto negativo de Letters of Intent sobre las prácticas laborales podría variar de -0.111 a -0.286 en gobiernos de izquierda.

En la parte inferior de la tabla 1, se observa que la severidad de las condiciones del FMI relativas al trabajo en Letters of Intent se asocia con factores políticos y económicos. ${ }^{48}$ Los coeficientes negativos y significativos en Left Party Power indican que hay una tendencia a que los países tengan condiciones prestatarias más suaves cuando los gobiernos y los partidos

\footnotetext{
48 Teri Caraway, Stephanie Rickard y Mark Anner, "International Negotiations and Domestic Politics: The Case of IMF Labor Market Conditionality".
} 


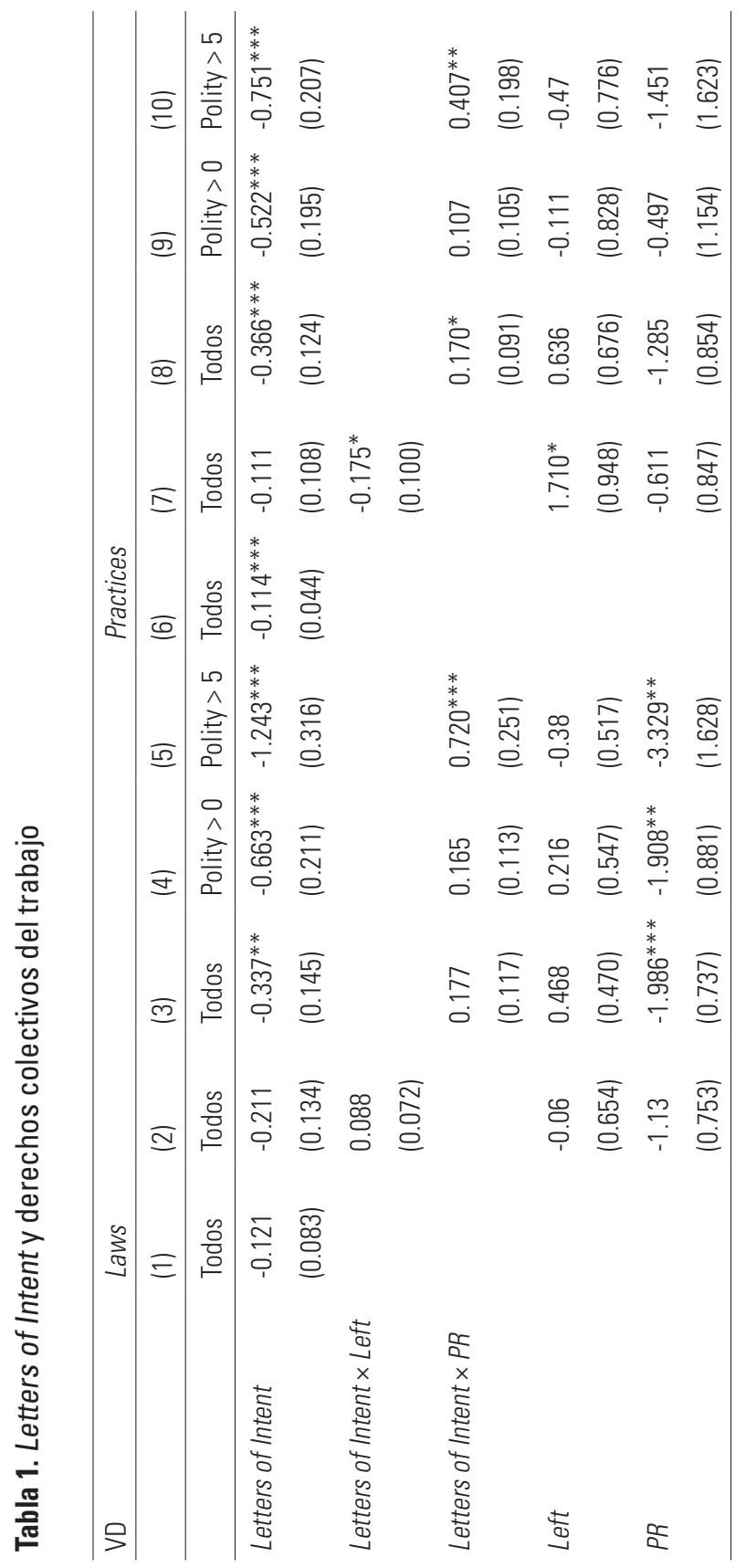




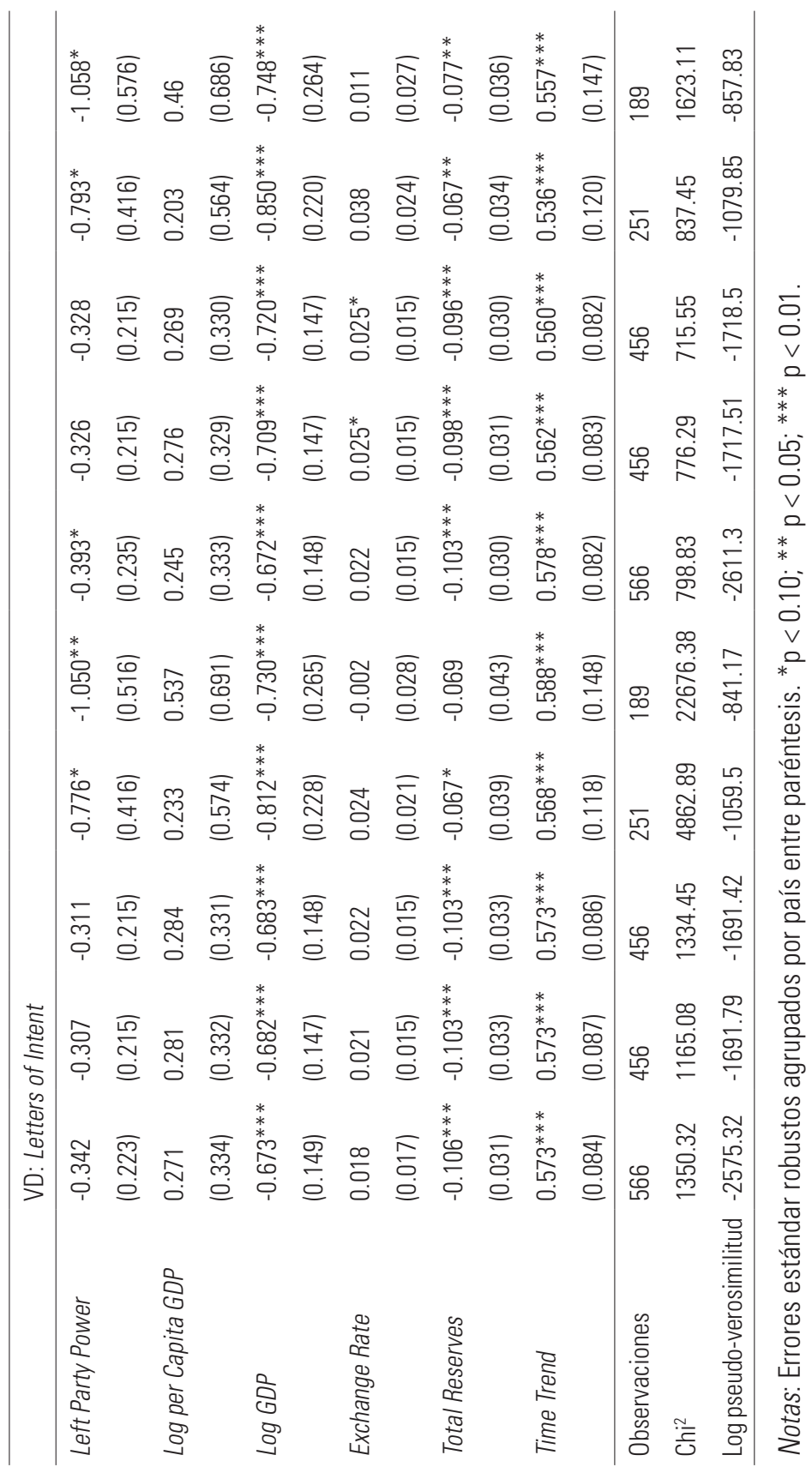


opositores refuerzan la representación política de izquierda. Los coeficientes en Log GDP, Total Reserves y Exchange Rate indican que las condiciones de los préstamos del FMI son mucho menos estrictas si un país presenta una economía grande, con un total de reservas abundantes y sin monedas sobrevaluadas. Los coeficientes positivos y significativos en Time Trends (tendencias temporales) señalan que tanto la cantidad como el alcance de las condiciones del FMI relativas al trabajo han aumentado con el tiempo. Si bien no se presentan en este trabajo, los coeficientes en UNSC y UNGA, no son significativos de forma constante, lo que niega los efectos de las variables geopolíticas en las condicionalidades del FMI asociadas a temas laborales.

En la tabla 2, estimamos nuevamente los efectos de las condiciones en el mercado laboral sobre los derechos colectivos del trabajo en otro tipo de documento de préstamo del FMI, Arrangement Letters. De acuerdo con Caraway et al., las cartas acuerdo pueden revestir mayor importancia para los países prestatarios, ya que en ellas se detallan las condiciones de reforma laboral que el FMI pretende implementar para su financiamiento. ${ }^{49}$ Los resultados de la tabla 2 respaldan nuestra hipótesis de que el nivel en que la severidad de las condiciones del FMI relativas al trabajo socava las leyes y las prácticas laborales se ve condicionado por los sistemas electorales. Los coeficientes en Arrangement Letters son negativos, mientras que sus términos de interacción con $P R$ son positivos en la mayor parte de los casos. En los modelos 3 y 8 se muestra que la RP de lista cerrada genera un cambio en los efectos marginales de Arrangement Letters de -0.277 a -0.105 para Labor Laws y de -0.163 a -0.085 para Labor Practices, respectivamente. Dichos resultados se mantienen firmes cuando analizamos, particularmente, las democracias estables (con puntajes Polity > 5), tal como se muestra en los modelos 5 y 10.

49 Ibid. 
Resulta interesante señalar que en las tablas 1 y 2 se muestra que los efectos condicionales de Arrangement Letters en los derechos colectivos del trabajo son de una magnitud mucho más considerable que los efectos condicionales de Letters of Intent si el modelo contempla las democracias estables. Estos resultados indican que las condiciones relativas al trabajo pretendidas por el FMI en Arrangement Letters producen efectos más perjudiciales tanto en las leyes como en las prácticas laborales que las propuestas en Letters of Intent por los países prestatarios. Los sistemas con RP de lista mitigan los impactos negativos de Arrangement Letters en las leyes y las prácticas laborales; sin embargo, su efecto produce un impacto mucho mayor al de Letters of Intent.

En las tablas 3 y 4 se muestran más resultados obtenidos mediante el uso de modelos de interacción de tres vías para las leyes, las prácticas y los puntajes originales de los derechos laborales propuestos por Mosley y Uno. ${ }^{50}$ En este trabajo, presentamos los resultados más importantes obtenidos de la ecuación de los derechos laborales, debido a que los resultados de las estimaciones para las condicionalidades asociadas a temas laborales en Letters of Intent y Arrangement Letters son similares a las presentadas en la parte inferior de las tablas 1 y 2. En la tabla 3, se muestra que la totalidad de Letters of Intent se asocia negativamente con los tres indicadores de los derechos colectivos del trabajo. Los términos de interacción multiplicativos de cada modelo también indican que la medida en que las Letters of Intent ejercen un efecto negativo sobre cada indicador de los derechos colectivos del trabajo depende de la configuración del partidismo de los gobiernos y de los sistemas electorales. En el modelo 1, el efecto marginal de Letters of Intent puede expresarse con la ecuación (1) que se encuentra a continuación.

$$
\begin{aligned}
& \text { (1) } \partial \text { Labor Laws/2Letters of Intent }=-0.783+0.576 \times P R \\
& +0.546 \times \text { Left }-0.441 \times P R \times L e f t
\end{aligned}
$$

50 Layna Mosley y Saika Uno, “Racing to the Bottom or Climbing to the Top? Economic Globalization and Collective Labor Rights". 


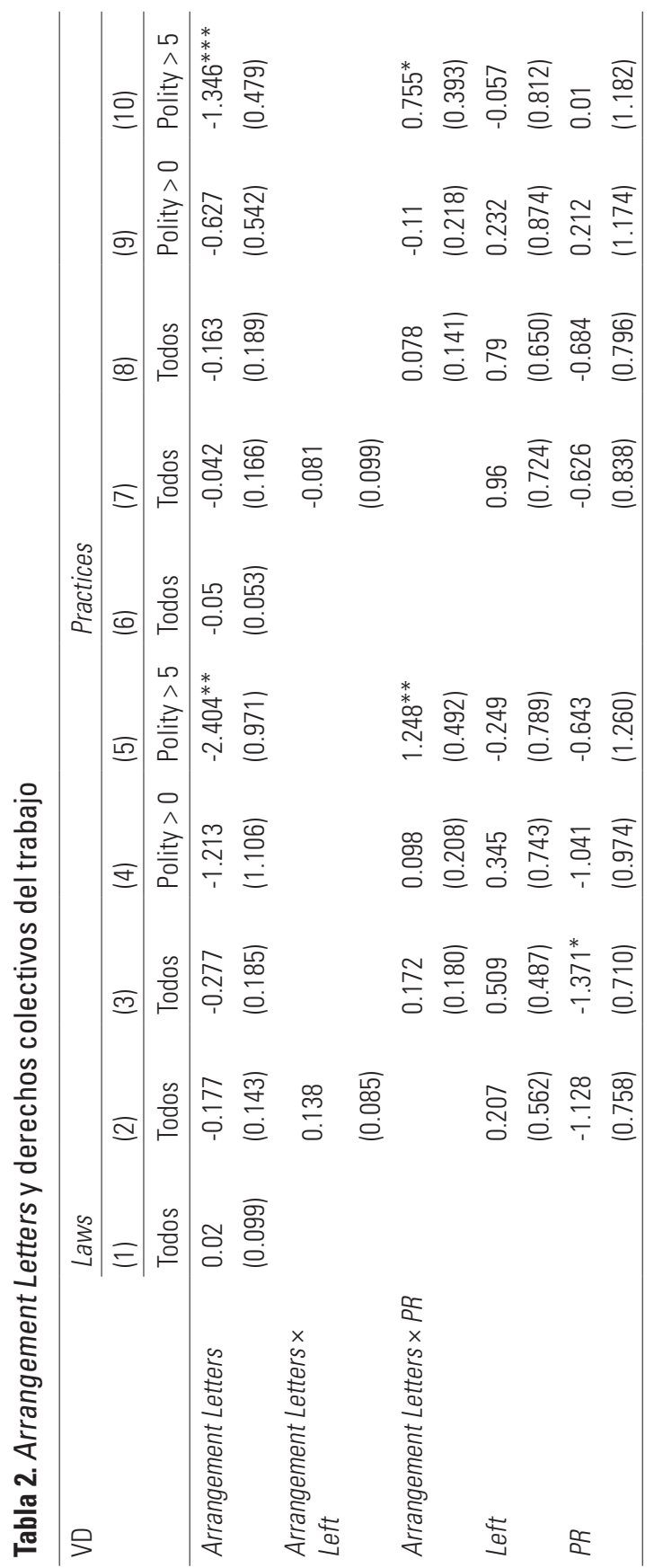




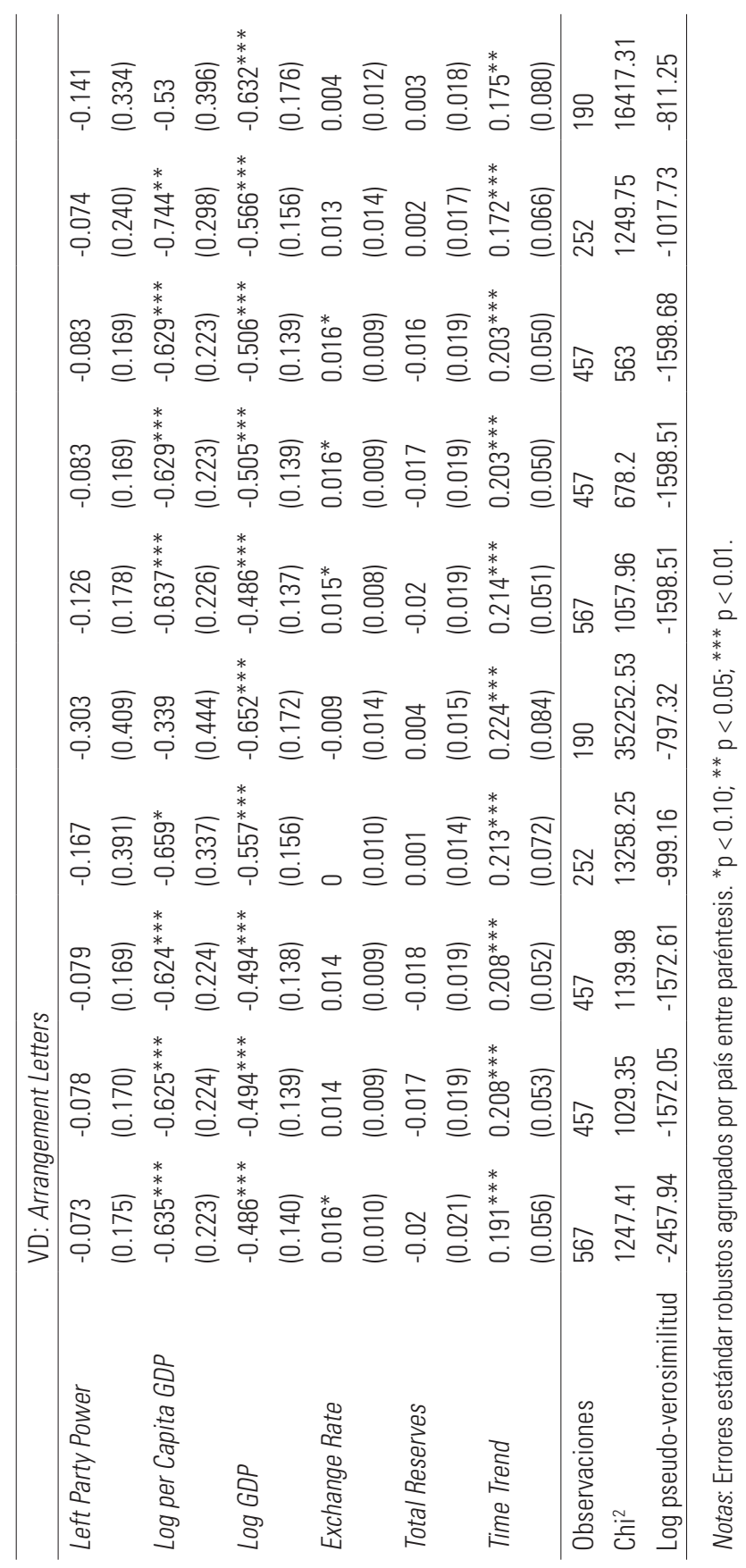




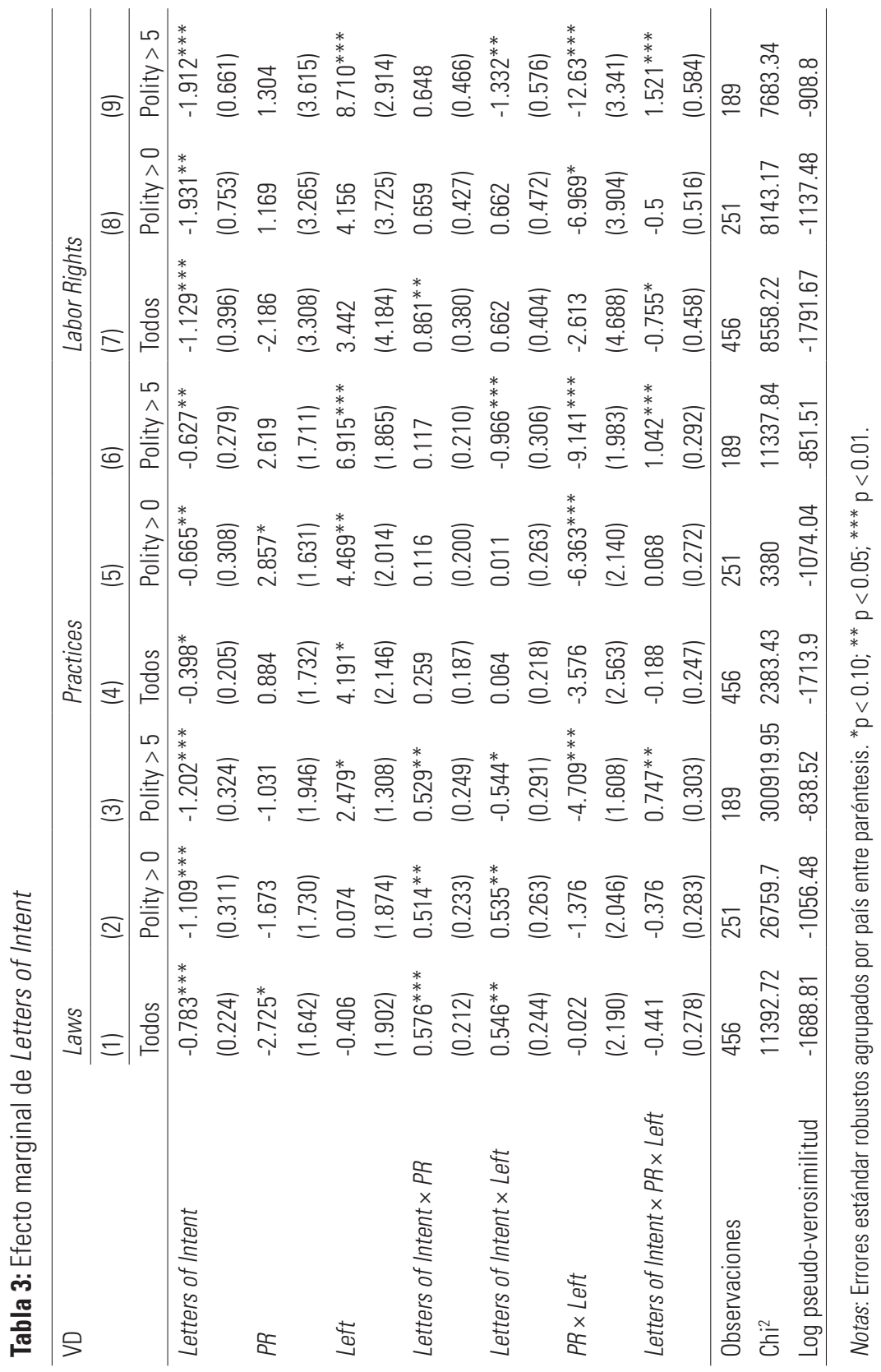




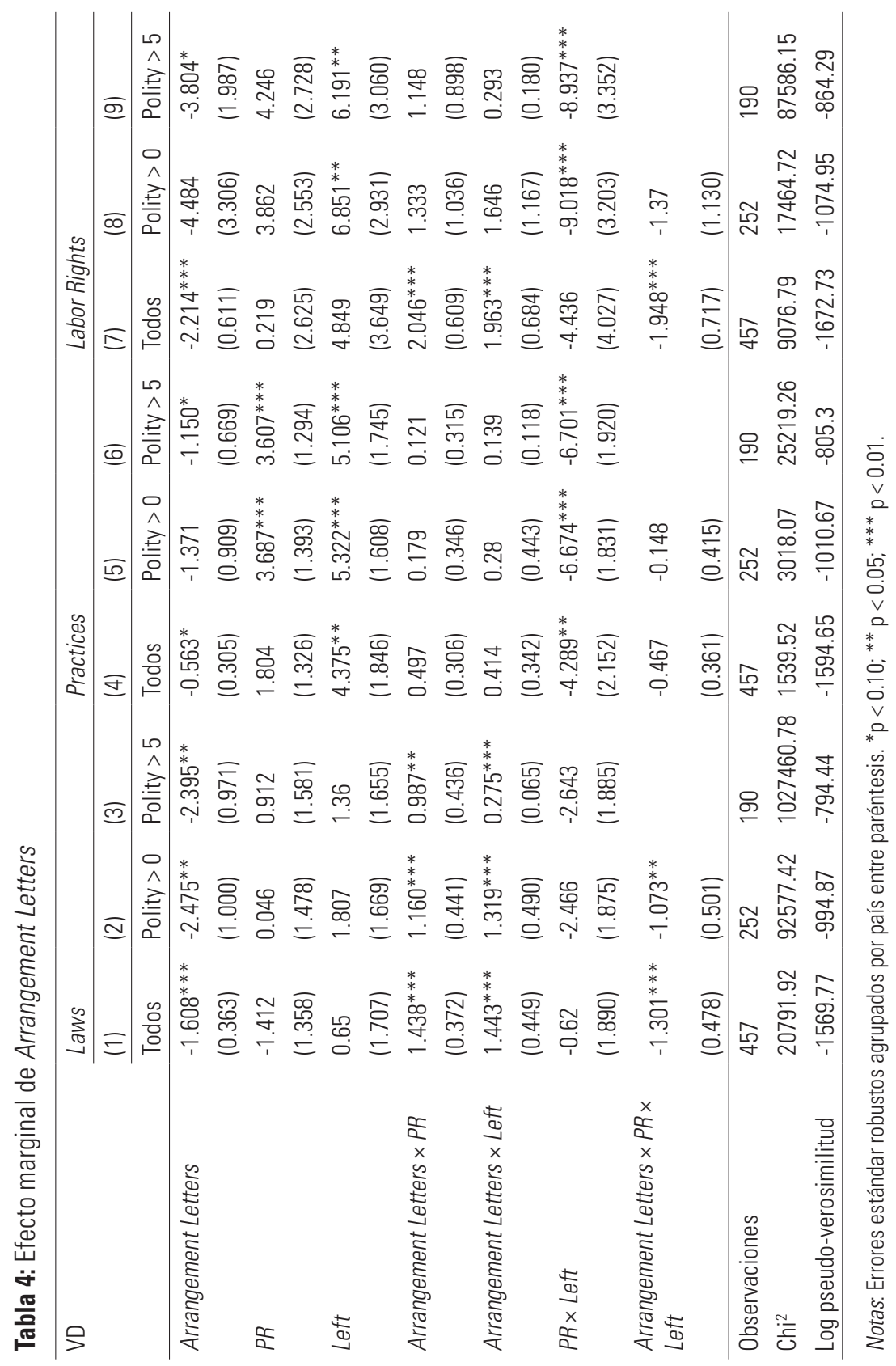


Debido a que tanto $P R$ como Left son variables ficticias codificadas con 0 o 1 , la ecuación (1) muestra que el efecto marginal de Letters of Intent será -0.783 , pero se modificará a -0.207 y -0.237 , respectivamente, ante la presencia de $P R$ o Left. Cuando un país está bajo un gobierno de izquierda dentro de un sistema con RP de lista cerrada, el efecto de Letters of Intent sobre las leyes se vuelve de -0.102. Los modelos restantes en la tabla 3 indican con certeza que la magnitud del efecto negativo de Letters of Intent sobre los derechos laborales alcanza su punto máximo cuando el país prestatario está liderado por un gobierno de derecha con sistemas mayoritarios, moderado por Left o $P R$, y se reduce al mínimo con un gobierno de izquierda moderado por Left y por $P R$. En particular, los resultados para las democracias consolidadas muestran que hay una mayor probabilidad de que los efectos de reducción de Letters of Intent sobre los indicadores de los derechos laborales puedan mitigarse mediante sistemas con RP de lista cerrada en lugar de gobiernos de izquierda.

En la tabla 4 se muestra, nuevamente, que es más probable reducir la magnitud de los impactos negativos de las condiciones del FMI relativas al trabajo sobre los derechos colectivos del trabajo mediante la combinación de gobiernos de izquierda y RP de lista cerrada. Excepto por los modelos 4 y 7, los resultados en la tabla 4 muestran, de forma consistente, que existen pocas probabilidades de que Arrangement Letters disminuya el nivel de respeto por los derechos laborales bajo gobiernos de izquierda, en un escenario con RP de lista cerrada. En particular, los resultados para las democracias establecidas (con puntajes Polity $>$ 5) indican que, independientemente del partidismo de los gobiernos, la medida en que las condiciones relativas al trabajo en Arrangement Letters socavan los derechos colectivos del trabajo es mucho menor con la RP de lista cerrada que lo que podría ser en otros casos. 


\section{Comprobación de la robustez de los resultados}

En la sección anterior se demuestra que hay una asociación significativamente negativa entre la intrusión de las condicionalidades relativas a temas laborales del FMI y el nivel de respeto de un país por los derechos colectivos del trabajo. Asimismo, se muestra que la magnitud de dichos efectos se encuentra condicionada por las instituciones electorales y la inclinación ideológica de los gobiernos.

Para comprobar la solidez de estos resultados, modificamos nuestras variables dependientes e independientes. En la tabla 5, se muestran los impactos de la participación del FMI en los tres indicadores de los derechos colectivos del trabajo. Se usó Years under IMF programs (años con programas del FMI), definida como la cantidad de años acumulados en que un país participó de los acuerdos con el FMI. Esta variable aumenta de forma anual si un país todavía se encuentra sujeto a acuerdos con condiciones concesionarias o no concesionarias en dicho año, de lo contrario se mantiene igual al registro del año anterior. Generamos Years under IMF Programs para el período 19802011 con los perfiles de los países disponibles en la Información de los Acuerdos de Préstamos del FMI (del inglés IMF Lending Arrangement Data). ${ }^{51}$ Determinamos el año 2011 como punto de final de esta variable, ya que también analizamos su impacto en los derechos de los trabajadores en la Base de Datos sobre Derechos Humanos de CIRI (del inglés CIRI Human Rights Database), ${ }^{52}$ tal como se muestra en la tabla 6.

Los resultados en la tabla 5 indican con certeza que la participación de un país en los programas del FMI tiende a socavar los niveles de respeto de dicho país por los derechos colectivos del trabajo, y que, ante la presencia de sistemas con RP de lista

\footnotetext{
51 http://www.imf.org/external/np/fin/tad/extarr1.aspx.

52 David L.Cingranelli, David L. Richards y K. Chad Clay, "The CIRI Human Rights Dataset", Versión 2014.04.14, http://www.humanrightsdata.com.
} 
cerrada, la magnitud de dichos efectos se modera en gran medida. ${ }^{53}$ En la tabla 6, la aplicación de las variables CIRI Workers' Rights (Derechos de los/as trabajadores/as según CIRI) y Years under IMF Programs aumenta la cantidad de observaciones a 3343 en 144 países para el período 1980-2011. Los resultados que se muestran en la tabla 6 indican con certeza que los escenarios con RP de lista cerrada mitigan significativamente los efectos negativos de los programas del FMI sobre los derechos laborales. Asimismo, dichos resultados se mantienen firmes en los distintos tamaños de muestra utilizados. En los modelos 1, 2 y 3 se contemplan todos los países desarrollados y en vías de desarrollo sobre los que hay datos disponibles. En este caso, los resultados muestran que la medida en la que Years under Program afecta los derechos de los/as trabajadores/as según CIRI alcanza su punto mínimo ante la presencia conjunta de las variables Left y $P R$ en democracias (con puntajes Polity $>0$ ) y democracias estables (con puntajes Polity $>5$ ). Las variables en los modelos 4, 5 y 6 solo se analizan para países en vías de desarrollo, mientras que en los modelos 7,8 y 9 se estiman para los países en vías de desarrollo (sin incluir a los países poscomunistas, de acuerdo con los hallazgos de estudios anteriores). ${ }^{54}$ Nuevamente, nuestros resultados demuestran que los efectos negativos de los programas del FMI se mitigan de forma significativa con la presencia de $P R$ y, especialmente, cuando se usan en forma conjunta $P R$ y $L e f t$, tal como se muestra en los modelos 6, 8 y 9 .

Finalmente, en la tabla 7 se analizan los efectos interactivos de los programas del FMI, el partidismo de los gobiernos y

\footnotetext{
53 Si bien las covariables de Years under IMF programs son mayormente similares a las de Letters of Intent y Arrangement Letters, agregamos otras variables de control que señalan la época posterior a la Guerra Fría y los antecedentes coloniales de los países prestatarios en relación con el Reino Unido, los EE. UU. y Francia, respectivamente.

54 Rodwan Abouharb y David L. Cingranelli, "IMF Programs and Human Rights, 1981-2003"; Brian Greenhill, Layna Mosley y Aseem Prakash, "Trade-based Diffusion of Labor Rights: A Panel Study, 1986-2002".
} 
Log District Magnitude (Log Magnitud del distrito), que indica la proporcionalidad de los sistemas electorales. Log District Magnitude equivale al logaritmo natural de la magnitud del distrito promedio de los escaños de la cámara baja. Por lo tanto, su valor es cero para los distritos uninominales con reglas de pluralidad, pero aumenta a medida que la magnitud del distrito es mayor a uno. ${ }^{55}$

En la tabla 7 se demuestra que Years under IMF Programs ejerce, de forma significativa, efectos negativos sobre las leyes, las prácticas y los derechos laborales de los países prestatarios. Excepto por lo reflejado en el modelo 6, nuestros hallazgos indican, de manera uniforme, que el nivel en el que los programas del FMI socavan los derechos colectivos del trabajo se vuelve mayor en los casos donde hay gobiernos de derecha con un valor de Log District Magnitude igual a cero, y que, tanto Left como Log District Magnitude, mitigan significativamente los impactos negativos de los programas del FMI sobre los derechos laborales.

55 Ronald Rogowski y Mark Andreas Kayser, "Majoritarian Electoral Systems and Consumer Power: Price-Level Evidence from the OECD Countries". 


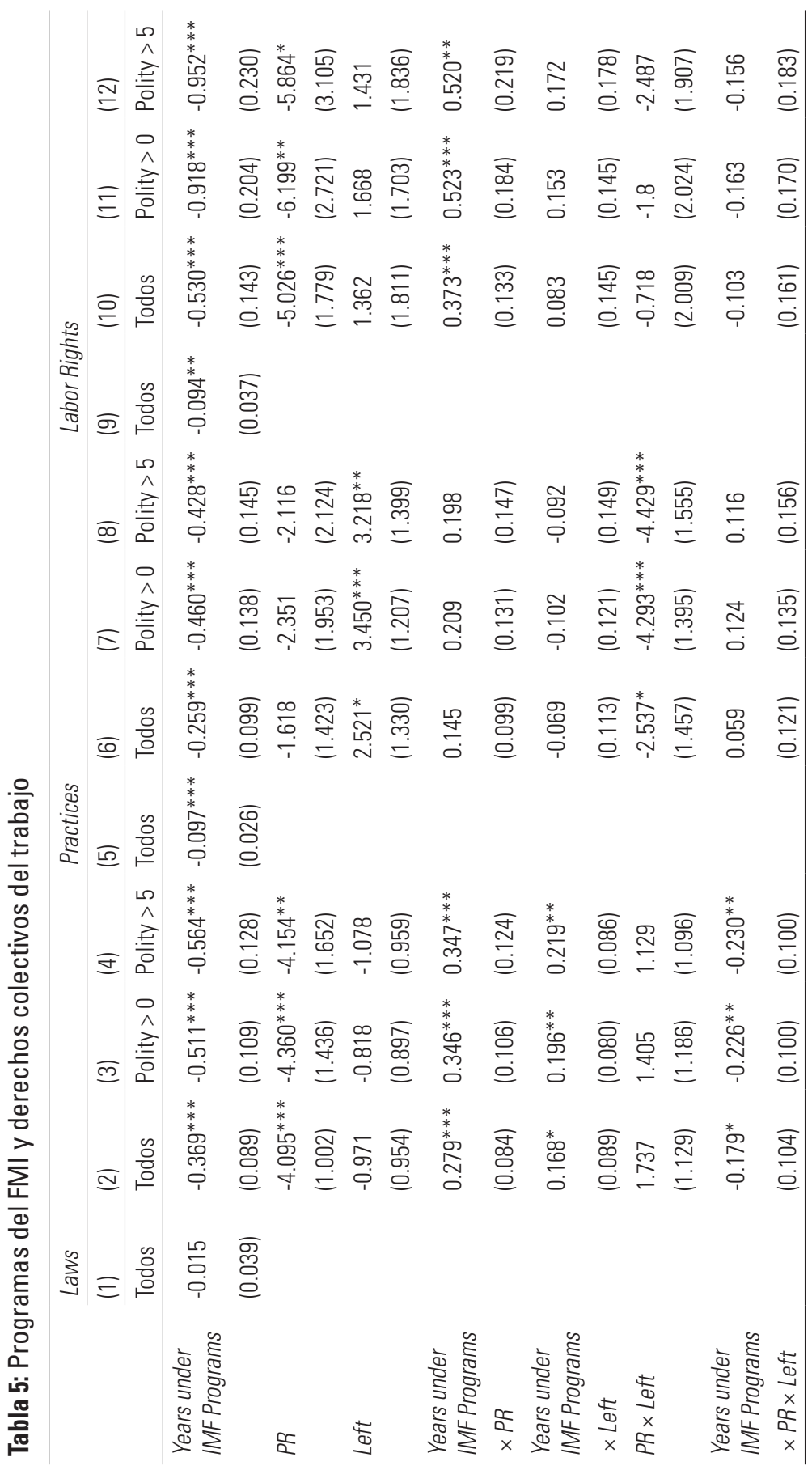




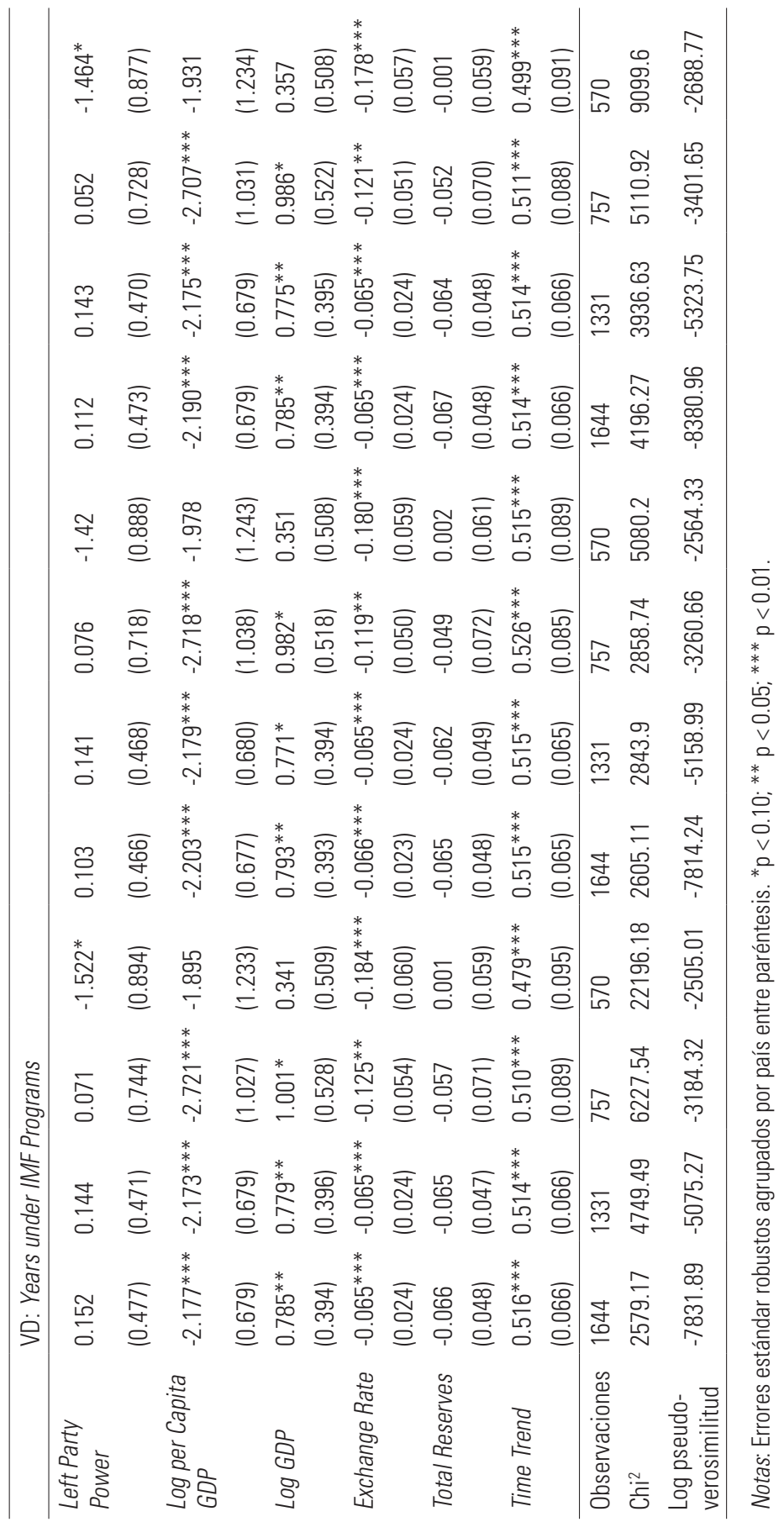




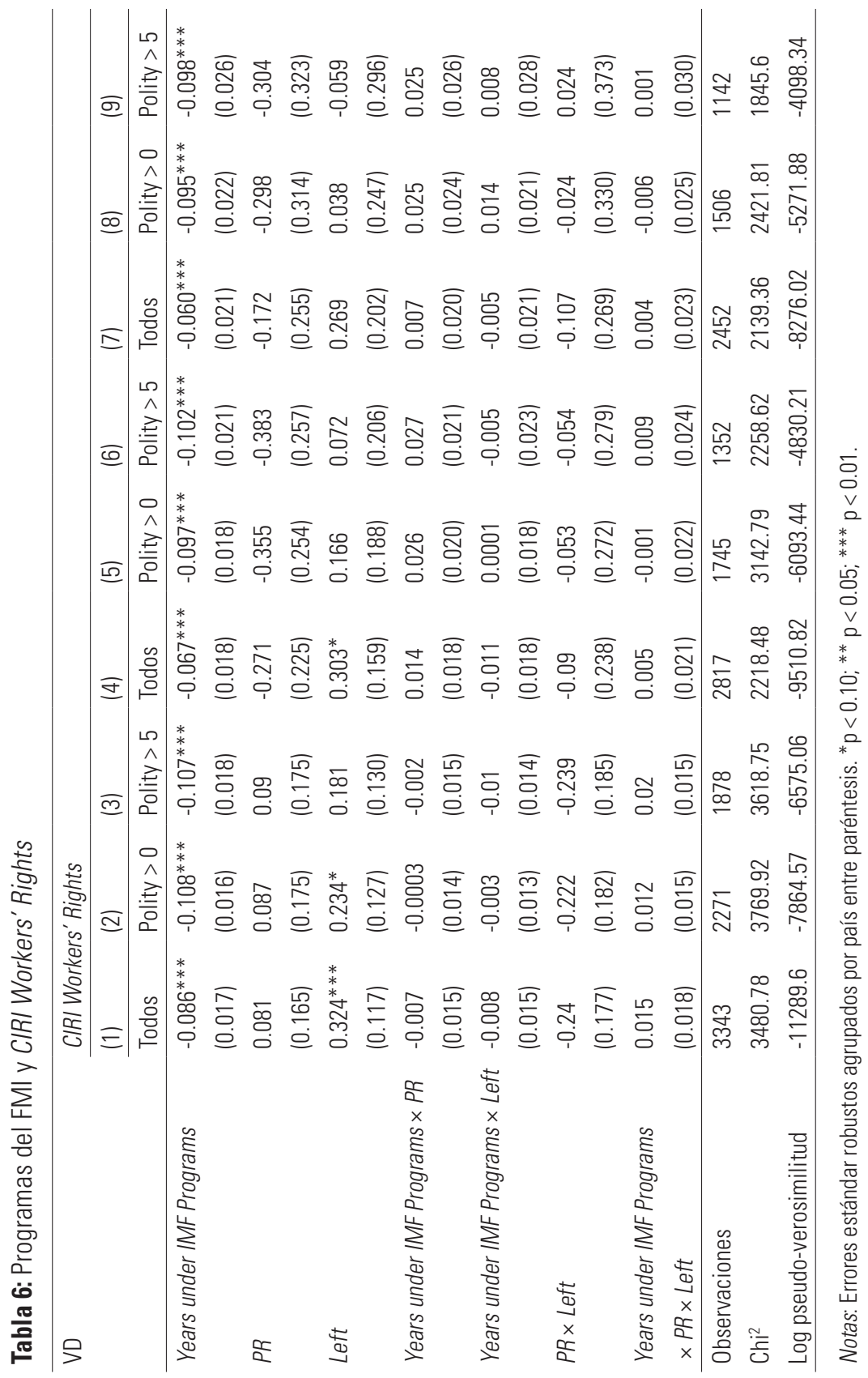




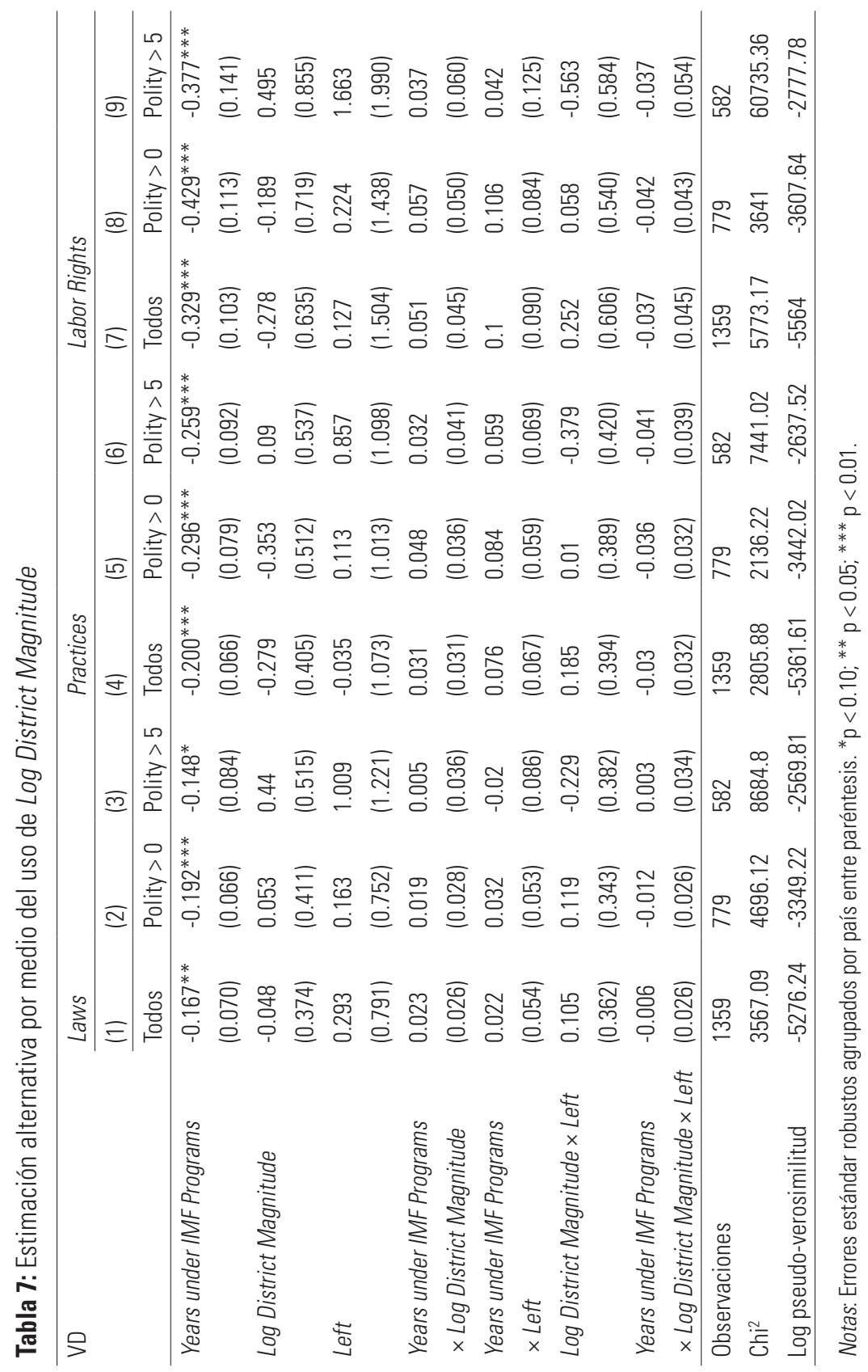


Fig. 1. Proporcionalidad y efectos marginales de los programas del FMI
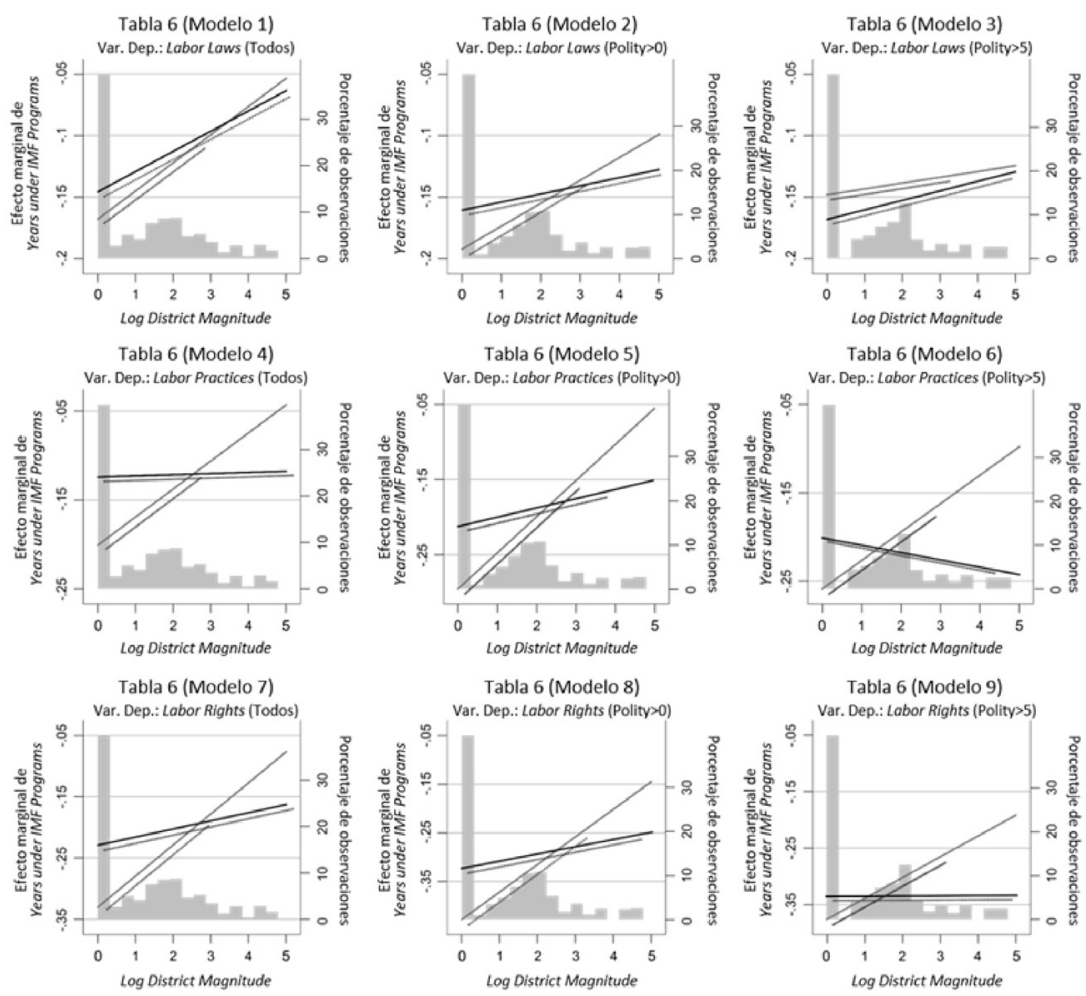

Dado que la relevancia estadística de los coeficientes de interacción multiplicativos en la tabla 6 solo tiene un valor indicativo, los efectos marginales de la condicionalidad del FMI en los derechos colectivos del trabajo se muestran de forma gráfica en la figura 1. Las dos líneas sólidas de cada gráfico indican los efectos marginales de Years under IMF Programs sobre los indicadores de los derechos laborales, condicionada por $\log$ District Magnitude y el partidismo de los gobiernos. Los cambios en los valores de Log District Magnitude se muestran en el eje horizontal, mientras que los efectos marginales de Years under IMF Programs durante gobiernos de centro-izquierda y 
gobiernos de derecha se destacan en negro y gris, respectivamente. Se agregan asteriscos para señalar los casos en que los coeficientes individuales para Years under IMF Programs son significativos a nivel del $90 \%$. En la figura 1 la curva positiva para los efectos marginales de Years under IMF Programs confirma claramente que el nivel en que los programas del FMI afectan negativamente las condiciones de los derechos laborales se reduce considerablemente a medida que aumenta la proporcionalidad de los sistemas electorales. Asimismo, en la figura 1 se demuestra que es posible que los gobiernos de centroizquierda mitiguen los efectos negativos de los programas del FMI sobre los derechos laborables. No obstante, estos efectos no siempre son consistentes.

\section{Conclusión}

Si bien en reiteradas ocasiones el FMI ha exigido un crecimiento más equilibrado e inclusivo, y ha puesto énfasis en ello, su materialización parece difícil de alcanzar. ${ }^{56}$ Por ejemplo, a principios de 2011, cuando el director gerente del FMI, Dominique Strauss-Kahn, y el entonces presidente del Banco Mundial, Robert Zoellick, se reunieron en Washington con una delegación de la Confederación Sindical Internacional (ITUC, por sus siglas en inglés), repitieron el discurso de que estas instituciones surgidas en Bretton Woods están comprometidas a "ampliar la distribución del crecimiento económico y ofrecer protección social". ${ }^{57}$ No obstante, más allá de las repetidas declaraciones de intención, la forma en que se tomarían medidas concretas para lograr los objetivos mencionados resulta incierta.

56 A modo de ejemplo, se puede consultar el número de diciembre de 2013 de la revista IMF Survey, disponible en https://www.imf.org/external/pubs/ft/survey/so/2013/pol121 213a.htm.

57 http://www.theguardian.com/global-development/poverty-matters/2011/jan/26/imf world-bank-recovery-workers-rights. 
Demostramos que, efectivamente, un análisis ampliamente aceptado de los efectos que el FMI y el Banco Mundial causan en los/as trabajadores/as pareciera sugerir lo contrario a lo que el FMI quiere escuchar. Es decir, que los programas y las condicionalidades del FMI tienden a ocasionar efectos significativamente perjudiciales en los derechos de los/as trabajadores/as en términos legales y prácticos. Estos efectos negativos pueden mitigarse únicamente si las circunstancias políticas en los países que participan de los programas son favorables a la representación política de los/as trabajadores/as, incluyendo gobiernos de izquierda y sistemas de representación proporcional. 